Témoigner Témoigner. Entre histoire et mémoire

Getuigen Revue pluridisciplinaire de la Fondation Auschwitz

$119 \mid 2014$

II y a 70 ans, Auschwitz. Retour sur Primo Levi

\title{
Catherine Perret, L'Enseignement de la torture, Réflexions sur Jean Améry
}

Yoann Sarrat

\section{Q OpenEdition}

1 Journals

Édition électronique

URL : https://journals.openedition.org/temoigner/1633

DOI : 10.4000/temoigner.1633

ISSN : 2506-6390

\section{Éditeur :}

Éditions du Centre d'études et de documentation Mémoire d'Auschwitz, Éditions Kimé

\section{Édition imprimée}

Date de publication : 31 décembre 2014

Pagination : 198-200

ISBN : 978-2-84174-688-0

ISSN : 2031-4183

Référence électronique

Yoann Sarrat, "Catherine Perret, L'Enseignement de la torture, Réflexions sur Jean Améry », Témoigner. Entre histoire et mémoire [En ligne], 119 | 2014, mis en ligne le 01 janvier 2016, consulté le

21 septembre 2021. URL : http://journals.openedition.org/temoigner/1633 ; DOI : https://doi.org/

10.4000/temoigner.1633 


\section{$\checkmark$ Librairie}

\section{GOEBBELS}

\section{Peter Longerich}

Traduit de l'allemand par Raymond Clarinard, Paris, Héloïse d'Ormesson, 2013, 880 p.

« Nous, Allemands, nous ne savons peut-être pas vivre. Mais pour mourir, nous sommes prodigieux. »

Goebbels, 1933.

e graphomane Goebbels a commencé à écrire quand, adolescent narcissique, il se prenait pour Schiller. En 1924, il se présente comme « communiste allemand » et toute sa vie il va se croire socialiste tout en acceptant, avec quelques hésitations vite oubliées, les avantages du pouvoir, voitures, palais, bateaux. Il est violemment opposé au christianisme et prévoit l'extermination des curés d'autant plus qu'il n'est pas loin de penser que « les nationaux-socialistes sont les seuls chrétiens authentiques. » Or, il sait la force des Églises et se marie religieusement, paye l'impôt d'Église et s'oppose à Adolf Wagner, le Gauleiter de Munich-Haute-Bavière, qui veut enlever le crucifix des murs des écoles. Mais peu importe les opinions de Goebbels. Il est prêt à en changer pour complaire à celui à qui il a décidé de vouer sa vie.

Goebbels est totalement fasciné par Hitler. Sa rencontre avec ce dernier qu'il compare au Christ est un signe du ciel. Il est dans une passion explicitement religieuse, voire amoureuse pour lui. Hitler joue de cette dépendance. Il intervient dans la vie privée de Goebbels pour lui indiquer s'il doit se marier ou s'il doit ou non divorcer. Les rapports de Hilter avec la femme de son ministre « à l'éducation du peuple et à la propagande », Magda, sont singulièrement ambigus et Longerich, évoquant les visites que fait la femme de Goebbels, seule, à la Chancellerie, parle même de « mariage à trois ». De toute manière, Hitler est un familier des époux Goebbels qui sont ses confidents. Quand Goebbels a « malheureusement » une fille comme premier enfant, Hitler le console : «Un garçon y aurait trop perdu à être comparé à son père. » Mais Hitler va aimer cette fille « comme sa propre enfant ».

Pourtant, malgré cette proximité de Goebbels avec son Führer, aucune grande décision politique n'est prise en concertation avec lui. Dès son accession au pouvoir, il est presque toujours mis devant le fait accompli sur lequel il doit pourtant adapter la propagande. Il est écarté des décisions d'intervention en Espagne. Il n'est averti ni de l'attaque contre la Pologne ni de celle contre l'Union soviétique qui le surprend totalement, il aurait été informé tardivement du début de la politique de l'euthanasie. Il n'est pas informé de la fin de la bataille de Koursk et Hitler tarde à lui dire la vérité sur le débarquement en Normandie. Hitler n'est même pas convaincu par le concept de Goebbels de la « guerre totale».

Cependant, Longerich pense qu’à la lecture de ses carnets, on peut supposer que Goebbels est informé des massacres commis dès le début de la guerre à l'Est.

En tout cas, malgré ses efforts pour contrôler ses relations personnelles avec le Führer, il n'arrive jamais à s'imposer comme le seul maitre de l'éducation et de la propagande. C'est Otto Dietrich qui est le chef du service de presse, pourtant un secteur essentiel de la propagande. Goebbels ne maîtrise pas davantage la propagande à l'étranger, entre les mains des services du ministère des Affaires étrangères de Joachim von Ribbentrop. Et il doit compter avec l'influence d'Alfred Rosenberg pour exposer la doctrine du national-socialisme en vue de l'éducation des masses.

Longerich, dans son livre sur Himmler, s'était contenté d'un bref paragraphe pour dire que le Reichsführer n'aurait pas été très haut placé si on lui avait appliquéles «critères raciaux » de la SS. Pour Goebbels, un de ces hommes qui déterminait la valeur des autres sur leur seule apparence, leur taille, la forme de leur crâne ou la couleur de leurs cheveux, Longerich évoque seulement sa «taille fluette». On peut comprendre que Goebbels ne s'entendait pas avec Rosenberg, le chantre des grands blonds nordiques dolichocéphales.

S'il n'a pas lui-même la capacité de la violence physique, il est doué pour la violence de la parole. On le voit souvent dans des procès, au Reichstag ou au Parlement de Prusse, hurler et inciter à la violence. C'est lui qui pousse à la « guerre totale » et qui encourage le lynchage des aviateurs alliés abattus sur le territoire du Reich. C'est surtout lui qui, devançant Hitler lui-même, pousse aux violences contre les Juifs, de la Nuit de Cristal jusqu'aux déportations. C'est lui quiveut 
marquer les Juifs et il est obsédé par le départ de tous les Juifs de Berlin. Il pense que ceux-ci, fauteurs de guerre doivent être éradiqués « d'une façon ou d'une autre ». Leur « destin est dur mais mérité » même si leur peine est « barbare ». Le 10 octobre 1941, il parle dans un discours de «l'anéantissement des Juifs ». Mais il comprend aussi que les nationaux-socialistes « ont brûlé leurs vaisseaux » et que la participation des Hongrois à la politique nazie contre les Juifs, les empêche de faire marche arrière et les lie au destin du Reich.

Les passages consacrés par Longerich au cinéma, le moyen le plus puissant de la propagande, sont cependant décevants. Il montre Goebbels plutôt bienveillant avec Fritz Lang, mais il émet des doutes sur le récit fait par Lang et accepté en général de sa rencontre avec Goebbels. Ce dernier ne s'entend pas avec Leni Riefenstahl. Il la trouve trop brouillonne et, évidemment, hystérique. Il admet qu’elle est douée mais « si seulement c'était un homme!» Ainsi c'est le parti et non le ministère de la propagande qui finance Triomphe de la Volonté. Longerich pourrait ajouter que c'est Bormann, sur les fonds de Hitler, qui finance pendant la guerre le film Tiefland où Leni Riefenstahl joue une danseuse tsigane.

Mais, dans l'ensemble, Goebbels reconnait l'échec de la politique culturelle nationale-socialiste. « Les films sont mauvais » et il convient aussi de la faiblesse de l'art et de la littérature.

Goebbels est conscient de l'inquiétude des Berlinois devant le risque de guerre et il note les réactions négatives de la population devant les déportations. Cependant, il ne mesure pas aussi bien que le SD (le Service de Sécurité) la dégradation de l'opinion allemande devant la tournure que prend la guerre, en particulier du fait des bombardements aériens. L'image de Hitler se déprécie dans l'esprit des Allemands alors que toute la propagande de Goebbels reposait sur l'exploitation du charisme hitlérien. Les nombreuses visites que fait Goebbels - contrairement à Hitler - dans les villes anéanties (Longerich indique systématiquement le nombre de morts dans chacune des cités détruites) ne parviennent pas à faire revenir l'opinion vers le national-socialisme et la personne de Hitler. Longerich conteste l'image de Goebbels, « virtuose » de la propagande.

Le livre de Longerich est un exemple de ce qui ne se fait guère en France à propos de l'histoire du national-socialisme : un livre clair, factuel, chronologique et divisé en chapitres relativement courts et faciles à lire. Déjà, l'éditeur avait cru bon de présenter la biographie de Himmler par Longerich à l'aide d'une formule publicitaire, sur la première page : « l'éclosion quotidienne d'un monstre ordinaire. » Pour la biographie de Goebbels, la citation de Die Welt : « une biographie définitive » est très inadaptée. Le mot « définitif » ne fait pas partie du langage historique (la publication de la biographie de Himmler en édition de poche évite heureusement ces inconvénients).

Jean-François Forges

\section{SHATTERZONE OF EMPIRES}

\author{
Omer Bartov, Eric D. Weitz (dir.)
}

Bloomington, Indiana University Press, 2013, 543 p.

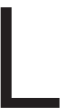

e titre Shatterzone of Empires annonce d'emblée l'approche choisie par Omer Bartov et Eric D. Weitz : la « zone de brisure » des quatre empires, dont les éclats formerontà l'issue de la Première Guerre mondiale les États-nations modernes, est une notion spatiale. Déployé sur plusieurs échelles - empires ou États, régions (Galicie, Haute-Silésie, Ruthénie carpatique), villes (Vilnius, Cracovie), bourgades (Buczacz), l'espace des borderlands devra être compris selon les différentes acceptions du terme : confins, marches, zones frontières. Rendre compte de la diversité ethnique, culturelle et religieuse de ces territoires ainsi que des violences de proximité qui s’y déroulent au cours du $\mathrm{XX}^{\mathrm{e}}$ siècle suppose donc d'intégrer les acquis du «tournant spatial » qui, depuis les années 1980, imprime de nouveaux cadres épistémologiques aux sciences humaines globalement et plus spécifiquement à l'historiographie et à l'histoire, discipline représentée majoritairement par les contributeurs du volume. L'idée d'une étude spécifique des confins a émergé depuis un certain temps déjà dans des travaux interdisciplinaires en Europe et aux États-Unis. En France, elle a notamment donné lieu, en 2005, à un numéro de Cultures d'Europe centrale, La destruction des confins (faisant suite à deux autres, Voyage dans les confins et Le Mythe des confins, publiés respectivement en 2003 et 2004), sous la direction de Delphine Bechtel et Xavier Galmiche. Par rapport à cette initiative pionnière, le 
volume que nous avons entre les mains élargit la problématique : l'intégration de l'Empire ottoman à la shatterzone a permis de construire une vision diachronique et comparative des violences génocidaires du XX siècle. Si l'ouvrage ne fait étonnamment aucune mention de ce travail (alors qu'Omer Bartov figurait parmi ses contributeurs), ses auteurs prennent en revanche une position critique, et ce, dès les premières pages de l'introduction, à l'égard d'une autre étude des violences qui adopte une approche spatiale : Bloodlands, Europe between Hitler and Stalin de Timothy Snyder paru en 2010 aux éditions Basic Books (New York). En effet, la violence dans les confins européens ne saurait être imputée seulement au conflit entre l'URSS et l'Allemagne nazie, car elle couvait en amont de ces régimes, souvent engendrée par la situation interne plutôt qu'imposée de l'extérieur. À la différence de Terres de sang, Shatterzone of Empires propose donc une exploration minutieuse à la fois des constructions imaginaires et symboliques et des configurations politiques et économiques.

Cette exploration des confins prend aussi en compte un autre virage historiographique, à savoir, le recours désormais possible à des sources considérées autrefois comme périphériques telles que discours, perceptions, imaginaires, mémoires subjectives, bref, éléments situés en marge des grands récits. La périphérie est ici bien davantage qu'une notion spatiale, elle se veut aussi donnée épistémologique : il s'agit de repenser, dans l'espace géographique et politique concerné, tout comme dans la hiérarchie des savoirs (entre la grande Histoire et la microhistoire, par exemple) le rapport entre centre et périphérie. Les confins, que même les pouvoirs les plus centralisés ou dictatoriaux ne contrôlent pas totalement, sont ainsiune métaphore d'approches nouvelles. Cette légitimation d'outils vis-àvis desquels l'historien se montre traditionnellement méfiant est toutefois soumise à une réflexion critique: ainsi l'article d'Omer Bartov, clôturant la quatrième partie sur les violences dans les confins, est basé sur des récits personnels relatant un génocide de proximité à Buczacz, en Galicie orientale, entre 1941-1944. Il en ressort que la prise en compte du témoignage personnel est nécessaire à l'écriture d'une histoire «tridimensionnelle », utilisant des outils de la microhistoire et de l'histoire du quotidien, et attentive à la complexité et aux contradictions qui disparaissent dès lors que l'on privilégie une analyse plus globale. L'étude spécifique de ces documents permet une vision de l'intérieur des communautés ainsi que des incursions dans le passé dont les historiens des violences font souvent l'économie. Toutefois, il ne s'agit pas de céder à l'illusion de saisir les logiques des violences « à la source » : une étude diachronique des confins depuis l'éveil des nationalismes de la fin du XVIII ${ }^{\mathrm{e}}$ siècle jusqu'à nos jours, suppose une approche dynamique des processus sociaux. On échappe à l'écueil théorique de la recherche de l'origine, mais aussi à l'interprétation des violences comme simple exacerbation des pratiques d'exclusion ou de ségrégation instaurées antérieurement; autant dire que tout est fait pour éviter une lecture mythologique des confins et de leur destruction.

Zones de liberté dans les empires, les périphéries deviennent facilement des lieux d'instabilité lors de l'éclatement de ceux-ci. Aussi, le passage à l'État-nation est-il questionné tout au long du volume en tant que modalité d'émergence d'une modernité qui, en modifiant en profondeur les configurations sociales, détruit l'équilibre des voisinages complexes. La construction de la figure problématique de l'autre (devenue progressivement objet d'exclusion voire d'extermination) est analysée dans la première partie de l'ouvrage (l'article de Larry Wolf) au prisme des imaginaires impériaux travaillés par l'identité, à partir de récits de voyage où l'on voit les Polonais par les yeux d'un Allemand (Fichte voyageant en 1791), les Allemands par les yeux d'un Polonais à la même époque (Niemcewicz). Ces constructions sont relayées par des mythes en œuvre dans des discours et des pratiques politiques, tel celui des confins orientaux de l'Allemagne perçus à la fois comme entrée dans un territoire menaçant et terre sauvage à civiliser. (Gregor Thum). Mais, avant de devenir une figure hostile, l'autre peut avoir été un « voisin invisible » comme les Juifs à Vilna-Wilno-Vilnius (Theodore R. Weeks) où, comme dans toute ville multiculturelle, la cohabitation ne repose pas nécessairement sur la tolérance et l'intérêt mutuel, mais aussi sur la négation de l'altérité par des populations considérant chacune le lieu comme le sien. Dans cette même partie, Dan Diner, s'interrogeant sur la situation des Juifs « entre empire et État-nation » depuis le milieu du XVIII ${ }^{\mathrm{e}}$ siècle, conclut à leur inadaptation au nouveau modèle. Si, individuellement, les Juifs sont des acteurs importants de la modernité européenne, en tant que communauté, ils suivent des schémas de la pré-modernité. Confrontées à des apories identitaires avec l'émergence d'une nouvelle conception de citoyenneté et d'identité nationale, ces communautés 
sont entraînées vers une catastrophe dontl'Holocauste sera la manifestation ultime.

C'est donc une multiplicité de facteurs, que l'on pourrait définir globalement comme l'avènement de la modernité, qui est convoquée pour comprendre le déclenchement des violences. Dans la deuxième partie consacrée aux espaces impériaux, Gary B. Cohen, puis Peter M. Judson proposent, sur l'exemple de l'Empire austro-hongrois, de se concentrer non sur la nature des mouvements nationalistes et le déclin des empires qui ne peuvent pas les satisfaire, mais sur le rapport entre l'État et la société. Les modalités d'entrée dans la modernité des populations des confins sont globalement repensées. Frithjof Benjamin Schenk montre notamment, en analysant des récits de voyage, comment le chemin de fer, qui permet à la population de l'Empire russe de découvrir la diversité ethnique et religieuse des confins (la zone de résidence juive, en l'occurrence), est aussi un outil aux mains de ceux qui cherchent à déstabiliser la situation politique (groupes terroristes ou auteurs de pogroms) : facteur d'intégration sociale a priori, le train l'est aussi de violence et de désintégration.

Élément original de l'ouvrage, les ambitions impériales sont envisagées - dans cette deuxième partie, mais aussi dans la troisième, consacrée à l'émergence des discours nationaux dans les confins, et la quatrième, portant spécifiquement sur les violences sous l'angle du colonialisme. Colonialisme turc (David Gound), manifesté par l'extermination des chrétiens assyriens, chaldéens et syriens de l'Empire ottoman, génocide mal connu à cause de la difficulté à attribuer une identité commune à ces populations qui, à la différence des Arméniens, se trouvaient hors de l'arène politique. Colonialisme russe (Peter Holquist), vu à travers les violences perpétrées par l'armée sur le territoire ottoman et en Perse du Nord (Ourmia et Asterabad). Rappelant les enjeux que constituait le Caucase dans le conflit russo-turque, l'article éclaire les pratiques militaires russes : contrôle du territoire, expéditions punitives, réquisitions massives, déplacements de populations, pratiques vouées à se perpétuer après la révolution dans le but d'intégrer les zones d'influence ou de conflit anciennes au nouvel État soviétique, puis à se généraliser au cours de la Seconde Guerre mondiale. Colonialisme allemand, enfin, qui a pour cible l'Empire ottoman et conduira à la complicité de l'Allemagne dans le génocide des Arméniens et des Assyriens (Eric D. Weitz). La pratique génocidaire a d'ailleurs été testée par les militaires allemands contre les Hereros et des Namaqua en Afrique du Sud-Ouest.

Si la Première Guerre mondiale offre un terrain d'observation particulièrement riche pour les violences imbriquées dans les confins, elle n'en constitue pas l'origine. Dans l'espace européen et limitrophe, persécutions et déportations ont déjà eu lieu pendant les guerres balkaniques de 1912-1913. La radicalisation du nouveau discours sur l'identité ottomane musulmane, l'exclusion des non musulmans de la communauté nationale qui conduiront, notamment, au génocide des Arméniens, doivent être comprises comme une de leurs conséquences (Eyal Ginio). L'émigration des populations chrétiennes (en l'occurrence, celle des Bulgares) largement encouragée par le gouvernement après la défaite est une étape vers l'idée de nettoyage ethnique. Elke Hartmann montre sur l'exemple des provinces kurdes et arméniennes isolées dans l'est de l'Asie Mineure, comment la fragmentation et la délégation du pouvoir dans les confins contribuent à l'escalade des violences, orchestrées par les États et réalisées souvent par des populations. Toujours dans le sillon des guerres balkaniques, on se penchera sur les massacres commis par des Grecs sur des civils bulgares (Keith Brown), ainsi que sur la valeur anthropologique des sources qui en attestent (lettres de soldats) contestées par les autorités grecques.

La perpétuation des violences d'une guerre à l'autre fait apparaître (Alexander V. Prusin) les pogroms de 1941, perpétrés par des Polonais et Ukrainiens sur leurs voisins juifs en Galicie occidentale, comme un héritage de la Première Guerre mondiale : pogroms et déportations que l'armée russe commet en 1914-1915, pillages par les locaux des maisons abandonnées. Le « syndrome de Jedwabne » est considéré, là encore, au prisme d'une modernisation qui intensifie les rivalités socio-économiques et politiques.

Les exécutions de prisonniers par le NKVD en Ukraine au moment de la retraite de l'armée rouge en 1941 ont servi, comme on le sait, de prétexte pour le déclenchement des pogroms anti-juifs. Sur l'exemple de Krakivski visti, quotidien ukrainien publié à Cracovie, John-Paul Himka suit la couverture médiatique internationale de ces crimes pour montrer l'ethnicisation à la fois des victimes et des criminels par les propagandes nazie et ukrainienne. À cet article fait écho celui de Kai Struve, dans la dernière partie de l'ouvrage portant sur rites, symbolisme et identité, qui propose une lecture des violences imbriquées staliniennes et nazies 
à partir d'investissements symboliques et identitaires de l'espace galicien et des bouleversements sociaux apportés par la révolution russe, à la suite desquels les Juifs, autrefois limités dans leurs droits, accèdent aux structures du nouveau pouvoir.

Cette dernière partie aborde des questions restées en marge des quatre autres, telles les frontières maritimes dans l'Adriatique Est (Pamela Ballinger); le renouveau artistique ukrainien et juif dans les confins apporté par la révolution russe et présentant une étonnante synthèse des aspirations nationales et internationales. On y trouve aussi l'étude d'un cas d'exception: celui de la coexistence interethnique sans violence dans la Ruthénie carpatique (Paul Robert Magosci). Enfin, la dernière contribution, de Philipp Ther, est consacrée aux régionalismes jusqu’à nos jours. L’exemple de la Haute Silésie, germanisée sous la domination allemande, polonisée de force après la guerre, permet ici d'envisager les régions non comme des lieux aux frontières définies, mais des objets de discours, donc des constructions symboliques en perpétuelle transformation.

On aurait aimé trouver dans ce panorama une étude de la soviétisation des confins qui suivit le partage de la Pologne et de la Roumanie entre l'URSS et l'Allemagne nazie en 1939 et se prolongea après la guerre ; manque tout particulièrement une étude sur la Bessarabie, annexée partiellement à l'URSS suite au pacte Germano-soviétique, puis reconquise par la Roumanie alliée de l'Allemagne nazie, dont l'un des territoires, la Transnistrie, devient alors une zone de déportation et d'extermination.

Enfin, si les logiques centripètes ou, au contraire, unificatrices sont évoquées à travers la notion de pangermanisme, on regrette l'absence d'une étude du panslavisme qui a joué un rôle tout aussi important notamment dans l'empire des Habsbourg. La guerre civile russe et sa conséquence, la guerre polono-soviétique, génératrices de pogroms à grande échelle, mériteraient également d'être abordées. Toutefois, le livre ne prétend pas à l'exhaustivité et vise surtout la mise en pratique de nouvelles approches : une réalisation avec laquelle il faudra désormais compter.

Luba Jurgenson

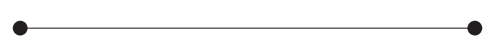

\section{L'IRRÉPARABLE. ITINÉRAIRES D'ARTISTES ET D'AMATEURS D'ART JUIFS, RÉFUGIÉS DU " TROISIEEME REICH » EN FRANCE}

Anne Grynberg, Johanna Linsler (dir)

Magdeburg, Veröffentlichungen der Koordinierungsstelle Magdeburg, Band 9, bearb. v. Andrea Baresel-Brand,

2012, 454 p.

$1 \mid$ rréparable » paraît de prime abord un terme inapproprié lorsqu'il s'agit d'évoquer le sort des œuvres d'art pillées et spoliées pendant la Seconde Guerre mondiale, de même que les caisses de tableaux renversées à la fin du Train, le film de John Frankenheimer sorti en 1964, ont quelque chose de dérisoire en regard des corps des otages assassinés dérisoire et presque obscène d'avoir sauvé des œuvres plutôt que des vies.

Cependant, le choix effectué par les auteurs de cet ouvrage d'étudier les trajectoires d'artistes et d'amateurs d'art juifs réfugiés en France pendant la guerre permet de lier le sort de ces hommes à celui de l'art qu'ils ont pratiqué ou collectionné. Ce lien explique que les sept artistes et amateurs évoqués dans cet ouvrage se soient tous exilés au moment de leur expulsion du monde de l'art allemand consécutif à la création de la Chambre de la Culture du Reich, sous l'autorité de Goebbels, le 22 septembre 1933, soit avant les lois de Nuremberg de septembre 1935. Le choix de la France et, plus précisément, de Paris s'explique quant à lui par le fait qu'à l'exception du jeune Horst Rosenthal, tous y avaient séjourné, souvent avant 1914, et entretenaient des liens personnels ou professionnels dans la capitale. Sept vies qui restituent un pan de l'histoire et du monde de l'art en France dans les années 1930, peu avant que ceux qui l'ont pu s'exilent à nouveau, cette fois vers les Amériques, comme l'a notamment montré Jean-Michel Palmier dans Weimar en exil. Le destin de l'émigration intellectuelle antinazie en Europe et aux États-Unis, paru en 1988.

Le critique et collectionneur Paul Westheim, dont Ines Rotermund-Reynard esquisse le parcours, fut de ceux-là. Il poursuivit à Paris ses activités journalistiques jusqu'en 1940 avant d'émigrer au Mexique l'an- 
née suivante grâce à l'Emergency Rescue Committee, le Centre américain de secours, dirigé en France par le journaliste Varian Fry, dont Anne Grynberg rappelle le rôle crucial dans l'introduction au volume. À l'opposé, le maître verrier et peintre abstrait Otto Freundlich qu'étudie dans ce volume Geneviève Dabien, ami de Kandinsky auquel il présenta Serge Poliakoff alors tout jeune peintre, ne put émigrer et il périt à Sobibor en 1943. La France ne le protégea pas ; Pablo Picasso ne put que payer les arriérés de loyer de son atelier et protéger ses œuvres jusqu’à la Libération. De celles-ci, les idéologues nazis firent l'emblème de l'«art dégénéré » et illustrèrent l'exposition de Munich de 1937 de La Grande tête que Freundlich avait réalisé en 1912; preuve qu'aux yeux des nazis la « dégénérescence » de l'art procédait de son « enjuivement».

Aussi le tort qu'on a fait à Westheim et Freundlich, comme à d'autres artistes parmi lesquels on peut citer ici Leo Maillet ou Jesekiel David Kirszenbaum dont Sandra Nagel et Johanna Linsler, respectivement, rappellent les parcours en tant qu'hommes et parce que Juifs, s'étend à leur propre activité artistique. Ils ont été persécutés à double titre : en tant que Juifs et en tant qu'artistes ou critiques d'art. En cela leur persécution relève du processus d'expropriation qui a constitué, pour Raul Hilberg, les prémisses de la destruction.

Pour ce qui a trait aux autres artistes, à ceux qui n'appartenaient pas aux avant-gardes, la conquête a brusquement fait passer leurs œuvres du rang d'objets de convoitise à celui de butins de guerre. Il n'est pas trop fort de dire que dès juillet 1940, Hitler, Göring, Goebbels, Rosenberg, Abetz et à leur suite des centaines de collectionneurs, d'historiens de l'art, de détectives privés et d'indicateurs français et allemands se sont rués sur l'art des collections privées juives de France avec ce mélange de dogmatisme et de gangstérisme qui a caractérisé l'entreprise de destruction et d'exploitation du III ${ }^{\mathrm{e}}$ Reich. Comme l'a montré Michel Rayssac dans L'Exode des musées, paru en 2007 auquel l'exposition Le Louvre pendant la guerre a fait suite en 2009, seuls les conservateurs des collections publiques françaises ont eu les moyens de résister, c'est-à-dire d'évacuer leurs collections puis de négocier pied à pied la préservation de leur intégrité.

L'action de Rose Valland se tient à la limite entre sphères publique et privée, et elle relève de la résistance. Affectée dès 1940 par le directeur du Louvre, Jacques Jaujard, au dépôt du Jeu de Paume, dans lequel les nazis ont entreposé et trié les œuvres spoliées, Val- land a recensé clandestinement toutes les œuvres qui ont transité par le Jeu de Paume et intégré après la guerre la Commission de récupération artistique qui a permis de retrouver et de rapatrier dans un premier temps 60000 des 100000 œuvres pillées et spoliées en France entre 1940 et 1944. Dans la difficile entreprise de recherche des sources sur le sujet, Anne Liskenne considère que le témoignage de Rose Valland qui a inspiré le film de Frankenheimer (paru en 1961, Le Front de l'art vient d'être réédité par la RMN) est l'un des ouvrages de première importance sur la question.

En note, Anne Liskenne rappelle que depuis la thèse de droit soutenue par Gérard Lyon-Caen en 1945, le terme de pillage désigne les actes de vol commis par les Allemands tandis que celui de spoliation correspond à la politique menée par l'État de Vichy à l'égard des biens artistiques comme des autres biens. En ce qui concerne les œuvres d'art, cette distinction montre avec quelle précocité le droit s'est emparé du sujet. Pourtant, en la matière, la notion de justice a peu à peu débordé celle de droit en recourant à un principe d'exceptionnalité analogue à celui applicable aux crimes contre l'humanité, et qui engage comme pour ces derniers un suspens de la temporalité qu'institue le droit.

Une notion comme celle de prescription indique en effet que le droit, entre autres « institutions », induit une mesure du temps humain et, ce faisant, produit une temporalité de la mémoire et de l'oubli. Le droit français, par exemple, écrit Anne Liskenne, « stipule que tout bien non réclamé revient au bout de trente ans à l'institution ou au particulier qui le détient. » Pourtant, ajoute-t-elle, « la loi n'a pas été appliquée à ces biens [les œuvres d'art], que ne touche, par conséquent, aucun délai de prescription et qui peuvent être à tout moment restitués aux familles concernées. » (p. 98) Dans l'avant-propos qu'ils ont écrit pour L'Irréparable, Jutta Limbach et Michel Jeannoutot rappellent qu'en Allemagne aussi des réparations peuvent être accordées en dehors d'un cadre strictement juridique, auquel se substituent de facto des principes relevant de l'éthique et de la morale. En pointant ainsi les limites juridictionnelles du droit, en soustrayant au droit le monopole de la délimitation de ce qui est juste, délimitation qu'il revient au politique d'établir une fois entamé le monopole du juridique, cette dérogation au temps judiciaire ouvre une temporalité alternative : celle de la mémoire à laquelle la société reconnaît une autorité équivalente à celle du droit, autrement dit une 
valeur jurisprudentielle non strictement juridique.

L’irréparable révèle cette atemporalité du préjudice subi et l'impuissance fondamentale du droit à le réparer. Il revient à l'histoire, telle qu'elle est pratiquée dans ce livre et telle que la fiction pourrait la narrer, de décrire comment ces artistes et amateurs ont été dépossédés de leurs œuvres et de leur art, c'est-à-dire d'une partie d'eux-mêmes, avant d'être à leur tour persécutés, descriptions qui sont comme les pièces d'un dossier sur lesquelles la mémoire, et non plus le droit, est fondée à se prononcer. La reproduction, en couverture de L'Irréparable, d'une autre tête d'Otto Freundlich, une tête de 1925 retrouvée à Berlin en 2010 au cours d'un chantier, une tête à demi brisée exposée avec ses manques et son fragment de crâne, porte cette mémoire et, au regard de l'histoire de l'art, cette reproduction atténue l'offense faite en 1937 à la Grande tête de 1912. I

Paul Bernard-Nouraud$$
\bullet
$$

\section{L'ENSEIGNEMENT DE LATORTURE, RÉFLEXIONS SUR JEAN AMÉRY}

\author{
Catherine Perret
}

Paris, Seuil, 2013, 207 p.

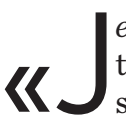
etzt passiert's » : « on y va ». Cette courte et terrible phrase, prononcée par le bourreau s'adressant à sa future victime, hantera à jamais Jean Améry, Hans Meyer, de son vrai nom, arrêté par la Gestapo en 1943 pour son activité dans la résistance belge puis torturé au fort de Breendonk avant d'être déporté à Auschwitz à cause de ses lointaines origines juives. Une vingtaine d'années après les faits, ce « juif de la catastrophe » (p. 158) délivrera un brillant témoignage multidimensionnel, Par-delà le crime et le châtiment, essai pour surmonter l'insurmontable (Jenseits von Schlud und Sühne. Bewältigungsversuche eines Überwältigten), sur sa condition d'être torturé et d'intellectuel déporté.

Près d'une cinquantaine d'années après la parution de cette ouvre au retentissement intellectuel puissant, Catherine Perret revient sur l'essai de Jean
Améry avec un brillant ouvrage : L'Enseignement de la torture, Réflexions sur Jean Améry, édité en 2013 aux éditions du Seuil. Ce dernier permet une importante relecture ainsi qu'une profonde réflexion sur l'intellectuel au destin tragique (Jean Améry, il faut le rappeler, s'est suicidé en 1978). Il invite à porter un regard sur ce corps torturé qui est le nôtre et sur ce «nous » qui se doit de résister encore, plus d'un demi-siècle plus tard. Au-delà de l'analyse du texte amérien, l'auteure rappelle que la torture persiste à être un grand problème d'actualité: c'est une méthode envisageable, une solution voire une nécessité dans certains cas, ce que critique vivement Catherine Perret dans sa préface en donnant d'importants exemples issus des événements de ce début de millénaire (notamment l'offensive post11 septembre du président Bush avec l'attribution de pouvoirs exceptionnels).

«[L]e mythe [nazi] a une scène: le camp d'extermination. Il a un rite : la torture. Un totem : le héros nazi. Et un tabou : le corps du juif dégénéré. » (p. 119)

Cette véritable expérience de « ruine des corps » (p. 79) constitue l'essentiel de la «philosophie » concentrationnaire. La torture devient une cérémonie, bien plus qu'un simple moyen, elle est un grand rituel que présente Catherine Perret dans son ouvrage, principalement dans le chapitre « Écrire le cri ». En tant qu' « essence spirituelle du Troisième Reich » (p. 86), la torture nazie reconfigure l'identité du bourreau par la construction progressive du martyre de sa victime, donc du corps de l'Autre. Constituant une sorte de négation de soi par anéantissement de l'Autre, Catherine Perret évoque l'autoréalisation meurtrière dont parle Jean Améry, un complexe définissant le sadisme de l'acte : la torture nazie s'inscrit alors dans un processus d'abnégation particulier. L' « être » est reconfiguré, et, comme le note Kafka : « le mot sein signifie en allemand à la fois : être là et être à » (p. 160), impliquant une double posture de l'être torturé, engagé dans cette relation avec le « je » bourreau : une amphibologie du verbe être.

La chair fait partie, pour le bourreau nazi en quête de l'autoréalisation héroïque, d'un système fantasmatique au centre de l'univers de la torture. Le corps de l'Autre devient le «ça » mourant, rejetant son « je » au profit du « je » du tortureur. Vient alors ce temps opposant diamétralement les deux instances de cette relation corps torturant corps torturé : le moment 
ultime où la victime devient «proie de la mort» (p. 116) (un quasi-mort, le pire étant ce « quasi »), animée d'un désir soudain de mourir plutôt que de subir, et où le bourreau se désintéresse de cette mort environnante puisqu'allant à l'encontre de son autoréalisation.

Catherine Perret réintroduit la notion de « mauvaiseté », proposée initialement par Thomas Mann, du bourreau nazi qui se doit d' « être grand en supportant la souffrance des autres. » (p. 119) Tout ceci fait partie d'une dialectique du corps souffrant au centre d'un rituel consistant en la vision autoréalisatrice de l'Autre souffrant, large réseau allant - Catherine Perret le rappelle - des gladiateurs antiques jusqu'à l'émergence des snuff movies, le besoin d'un visionnement de la souffrance corporelle de l'Autre devient expérience d'autoréalisation malsaine et sadique. La souffrance du corps torturé par le nazi est décrite comme une sorte de «parodie » de la transsubstantiation où la chair du torturé reconfigure l'identité du tortureur. Le corps torturé devient un « corps total » : «le corps torturé par le nazi n'est pas le corps du torturé. C'est notre corps. » (p. 9) La chair désigne alors le fantasme (le corps torturé serait personnification du fantasme d'autoréalisation).

Dans cette «torture-célébration » (p. 119), le nazisme considère les Juifs comme des stück (pièces), l’idéologie nazie réside dans la soustraction progressive de ces pièces - «l'extase du chiffre est la marque et l'extermination le nom » (p.97) - engagée dans un large processus relatif à l'univers concentrationnaire : avec, en autres, la désindividuation des corps par la nudité, l'anéantissement du nom propre et son remplacement par un matricule, « la destruction des imagos du moi par l'abolition des rites mortuaires » (p. 61), le tout visant à démantibuler progressivement l'identité de la persona. À cela s'ajoutent la volonté d'entassement et d'enchevêtrement des stück dans les chambres à gaz (conglobation de corps mourants), dans les trains, dans les fosses de cadavres, et la création de figures relatives à l'univers concentrationnaire de corps de souffrance exacerbée : le musulman (cet être qui n'est pas vivant, mais qui n'est pas tout à fait mort non plus, qui tombe sur place, pour mourir, dans un ultime mouvement transitoire, dans la position de la prière du musulman) ou le membre du Sonderkommando (chargé de « préparer » les autres déportés à la chambre à gaz et du nettoyage de celle-ci après gazage au Zyklon B).

L'écriture de la torture est une écriture de l'acmé, une expérience du cri scriptible. Ce cri est celui du corps torturé. L'acmé du rituel sadique est le terrible instant de l'aveu, cet instant où la chair se fait Verbe, même insensé (Améry s'attribue des actes qu'il n'a pas commis), moment où une « ombre [...] s'échappe du néant » (p. 132), ombre qui est le Moi. Ainsi, « l'essai d'Améry rejoue le sacrifice dont il fut l'objet » (p. 117), il élucide, dans la langue du bourreau, l'acte de sortie du Moi hors du corps torturé. Catherine Perret définit l'être selon une relation tripartite impliquant le sich (en soi), le selbst (pour soi), le der Nächste (le prochain) : la torture sacralise, dans l'instant culminant du rituel, un anéantissement du prochain, der Nächste, par le cri.

Ce moment du cri viscéral de la victime est une sorte d'irrintzina ultime : un cri absolu, qui expulse le moi hors du corps. Il s'agit alors d'entendre ce sur-cri paroxystique de l'Autre torturé pour sacraliser l'anéantissement. Freud parle de la « fonction primordiale du cri dans l'ontogenèse de l'individu. » (p. 121) Ce cri, émanant de la « chair hurlante » (p. 121), est une sorte de jouissance pour le corps-tortureur et un exact inverse pour le corps torturé: il est l'élément culminant d'une séparation nette dans la douleur ritualisée.

C'est lors de cette acmé du rituel, ce moment de négation totale et absolue de la victime par son bourreau, que Jean Améry fait le constat d'une expérience de l'indicible, où un sentiment parcourt l'ensemble du corps endolori, quasi-proie de la mort : « le sentiment d'être devenu à ce point étranger au monde qu'aucune communication ultérieure avec les hommes ne pourra jamais le compenser. » (p. 133)

Il est devenu «la chair hallucinée» (p. 134), le corps du trauma, une « corporalité mouvante et composite » (p. 135). Il est un corps qui se retire du monde à cause d'une transgression liée à la torture : celle de la peau comme écran supposément inviolable du corps-moi. Ce contact transgressif de l'épiderme est une attaque de la corporéité.

Par ailleurs, Catherine Perret revient sur le lien unissant l'œuvre d'Elias Canetti, Masse et Puissance et celle de Jean Améry. L'isotopie de la corporéité (et l'ensemble de ses attributs et système métonymique inspectés dans les essais) est au cœur de leurs réflexions. Il est ainsi question de cette expérience du moi freudien développé par le toucher réfléchissant (le contact de sa propre peau pour se sentir en tant qu'Être). Selon Catherine Perret, les deux auteurs convoquent deux modalités complémentaires du corps : « la phobie du contact » (« l'interdit du toucher ») et le « cristal de masse », « ce noyau à partir duquel la masse se multiplie 
par pure énergie autoproductrice. » (p. 142)

La peau devient lieu du trauma, l'espace de la transgression par laquelle le bourreau sactive en tant que corps-tortureur et démarre son rituel d'autoréalisation. Elle est ce qui sépare le dedans du dehors, son anéantissement constitue un viol de l'espace intérieur qui, a contrario du corps déporté, est un espace où la liberté est possible. Jean Améry rappelle que son identité se décompose en « je » multiple (l'intellectuel, le résistant communiste, le déporté, le Juif) mais qu’il n'existe qu'un seul corps torturé. La peau est cette surface pénétrée par effraction par ce qui devient alors l'extension du corps-tortureur : l'outil, l'objet de douleur. Les différents rites de déshumanisation du déporté passent, et Catherine Perret l'explique bien, par le contact et la transgression permanente de la peau par le nazi : c'est le marquage direct, à l'entrée du camp, du bras par le tatouage (et les chiffres, que l'on retrouve encore), c'est cette volonté permanente de raser, de nettoyer, etc. " Améry retrouve alors ce qui, dans la langue, fait de la peau la métonymie de la vie par opposition à la survie. » (p. 144) C'est par cette transgression ultime de la peau de l'Autre que la chair devient Verbe : la pénétration douloureuse de la peau sous l'effet de la torture permet le vomissement des paroles fausses.

L'umstülpen active un grand processus d'inversion et de retournement: au cours du rituel, la peau est non seulement manipulée, lacérée, déchirée, trouée, mais elle est aussi complètement retournée, comme celle d'un gibier, au point que la victime se trouve comme expulsée de son intimité, elle « habite une doublure vide» (p.146), retourner la peau c'est retourner le corps de la victime. Donc, en quelque sorte, faire sortir le dedans, violer l'intimité de la corporéité. L'être devient destructible, sa peau ne protège plus son corps et son Moi. La corporéité est attaquée et le stück devient viande, chair de la transsubstantiation autoréalisatrice. La honte devient ce point ultime de connaissance d'une frontière violemment transgressée qui ne devrait pas l'être, point d'acmé de l'expérience du corps torturé et processus d'autotorture. « Celui qui fut torturé reste torturé » (p. 143) : vient ensuite le temps du « choc de l'abandon » (p. 144) post-déportation, le constat de l'hypocrisie de la dénazification, alors, la torture se métamorphose. Le « nous » devient imprononçable, il n'existe plus.

«La torture est le cœur d'un rituel plus vaste dont le centre est la peau, dont le Lager est la scène, et dont le public allemand et européen est le spectateur horrifié et consentant. » (p. 129)

Plus loin, Catherine Perret interroge le concept du «Moi-Peau » (p. 162) de Didier Anzieu à la lumière des réflexions amériennes. Le «Moi-Peau » semble anéanti dans et par la torture, le Moi est privé d'un soutien extérieur et de ses défenses internes dans le même temps. Le «Moi-Peau » devient le « Nous-Peau » dans la réflexion de l'auteure, partant de l'excipit du texte d'Améry, où le «je » et le «nous » se côtoient enfin dans la même phrase. Vingt-deux ans après cette condition d'infans (« celui qui ne parle pas ») - où le silence du nouveau-né post-Shoah s'oppose au cri viscéral du torturé - le « nous », qualifié d'ancestral et « résonnant dans le Moi » (p. 174) par Catherine Perret, ressurgit enfin, il peut dire l'indicible, le procès d'Auschwitz (comme faisant partie d'un savoir d'un bien commun) reconditionne alors le «je ». Un « Nous-Peau », donc, peau d'un bien (Eigentum) commun, « qui résiste, quand ça résiste. » (p. 175)

À noter que le post-scriptum de l'essai de Catherine Perret s'achève sur une citation de Kafka issue de l'excipit de Le procès, l’image finale est donc celle du regard ultime d'une figure-martyr, être épiphanique au cœur transpercé, incarnation du sondage de « la condition profonde de la victime » de Jean Améry, parmi les plus importantes de la littérature, regard qui est aussi le nôtre, ce K. est aussi notre initiale... I

Yoann Sarrat

\section{QUAND ULYSSE REVIENT A TRIESTE}

\section{Boris Pahor}

Traduit du slovène par Jure Kozamernik, Paris, Pierre-Guillaume de Roux, 2013, 329 p.

l ne se passe apparemment pas grand'chose dans ce roman de Boris Pahor. Septembre 1943, Rudi, le héros, un jeune soldat slovène sous uniforme italien, vient d'apprendre que les troupes sous commandement italien doivent être enrôlées dans les forces allemandes pour combattre les partisans. Comme nombre de ses camarades, il a décidé de déserter. Il échappe aux 
Allemands en gare de Trieste, puis rejoint à pied un village du Karst proche de son bourg natal. Rejoindrat-il les maquis dans la montagne, ou la Résistance qui s'organise à Trieste? La fin du roman nous le montre descendant vers Trieste. Aucune autre action n'a eu lieu, sinon les réflexions du héros.

Malgré cette apparence presque lisse, le roman est porteur d'informations historiques qui donnent à réfléchir. Les rencontres de Rudi avec de vieux villageois sont l'occasion d'évoquer, dans leurs souvenirs, les débuts du fascisme et de la lutte anti-fasciste, mais aussi la composante nationaliste slovène : la haine de l'Italie, pouvoir dominant qui dévalorise la culture slovène et interdit l'enseignement de la langue, n'est pas pour rien dans l'antifascisme de certains. Le mépris italien pour les Slovènes est intériorisé par plusieurs personnages, comme Vida, qui a un amoureux italien et ne rêve que d'aller vivre en ville. Et pour s'y opposer, le héros - ou l'auteur - de retour dans son pays natal, tel Ulysse, fait l'éloge de la nature rude du Karst, qui façonne des hommes et des femmes à son image. Pour un lecteur français, un tel éloge a des connotations fâcheusement vichystes, mais il faut le nuancer : d'abord parce que la « nature » du Karst est mise au service d'une volonté de résistance à l'oppression, et surtout, parce qu'il n'y a pas d'opposition entre ville et campagne. Pas d'équivalent de l'affreux slogan « la terre ne ment pas », puisque le héros, au final, choisit d'aller lutter en ville.

Le lecteur qui voudrait connaître Trieste serait un peu frustré de cette lecture, puisque la ville demeure hors champ. La thématique homérique annoncée dans le titre reste en filigrane. Et l'on peut être plus ou moins sensible à l'analyse psychologique du personnage central, à ses hésitations amoureuses entre Vida la frivole et Majda l'insurgée, ou même aux scènes très vivantes qui touchent au genre du « roman de guerre » (la rafle en gare de Trieste, le silence des deux clandestins dans le tramway.) Mais l’intérêt du livre réside surtout dans cette affirmation du droit des minorités à une existence linguistique et culturelle - affirmation qui exige d'être examinée de près, pour éviter tout risque de confusion avec un quelconque communautarisme.

Anne Roche

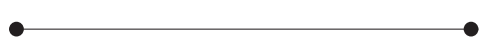

\section{TERRITOIRES DE MÉMOIRE. L'ÉCRITURE POÉTIQUE Â L'ÉPREUVE DE LA VIOLENCE HISTORIQUE}

\author{
Lucie Taïeb \\ Paris, Classiques Garnier, "Perspectives comparatistes », \\ 2012, 423 p.
}

ucie Taïeb nous présente dans Territoires de mémoire une recherche inspirée par un texte d'Elie Wiesel : « Comment fait-on pour faire du langage un refuge plutôt qu'une prison, une auberge plutôt qu'un cimetière? » Dans une perspective comparatiste passionnante, elle rassemble trois auteurs aussi disparates et à l'esthétique aussi dissemblable que le poète argentin Juan Gelman, l'écrivain français Edmond Jabès et la poétesse allemande Nelly Sachs. Des poèmes en espagnol (Gelman, De palabra, 1994) et en allemand (Sachs, 5 recueils), un ouvrage en français (Jabès, Le Livre des questions I, 1963) : pour comparer les textes et faire participer le lecteur à cette aventure comparatiste, il a fallu des traductions en français, notamment de poèmes de De palabra, de Fahrt ins Staublose (1961) et de Teile dich der Nacht (1971), deux ouvrages de Sachs qui n'avaient pas été traduits. Dans les cas où il n'existe pas de traduction, l'auteure nous en fournit de sa propre main. Cependant tous les extraits sont aussi présentés avec leur texte source. L’auteure a donc elle-même été soumise à l'épreuve de la traduction dans un essai sur l'épreuve de la violence historique. Nous sommes face à une étude multilingue où résonnent des références à Blanchot, Celan, Mechonnic, Bachmann, Buber, Scholem et tant d'autres grands penseurs et poètes germanophones et francophones du XX ${ }^{\mathrm{e}}$ siècle. Le cadre théorique est complété par des études anglo-saxonnes récentes des Holocaust Studies et par des ouvrages d'Imre Kertesz, de Primo Levi, de Victor Klemperer et d'autres auteurs, qui évoquent la mémoire traumatisante et qui forment une caisse de résonance pour les textes de Gelman, Sachs et Jabès.

Les œuvres de Sachs et de Jabès sont consacrées à la mémoire de la Shoah, celle de Juan Gelman est dédiée à la mémoire de la dictature argentine. Le projet ne vise pas à une comparaison de la Shoah et de la dictature argentine, mais à lire ensemble des œuvres 
qui traitent de ces événements distincts. Taïeb étudie la manière dont l'existence des trois poètes a été marquée par ces événements. Juan Gelman (1930), fils de Juifs ukrainiens émigrés en Argentine à la fin des années 1920, s'exile dès 1975 pour échapper au meurtre programmé par la junte militaire de toutes les personnes jugées « subversives ». Dans ses poèmes il s'adresse à son fils « disparu » en Argentine. Chez Nelly Sachs (1881-1970), née au sein d'une famille juive allemande et qui se réfugie à Stockholm grâce à Selma Lagerlöf, l'accent est porté sur la perte du peuple juif dont la poétesse veut porter le deuil et transmettre la mémoire de son extermination. Grâce à une lecture comparatiste, Taïeb réussit à établir un lien entre la poésie de Sachs et de Gelman par exemple dans leur rapport au divin et à l'exil. Né au Caire dans une famille juive francophone, Edmond Jabès (1912-1991) doit quitter son pays natal en 1956 à cause de ses origines. Dans son œuvre, il témoigne de l'horreur de la Seconde Guerre mondiale et de l'extermination du peuple juif. Selon Jabès, la Shoah fait désormais partie de l'héritage de l'Occident, « héritage à partir duquel il nous faut penser nos relations avec les autres » (p. 398). Dans la lecture de Taïeb, il se rapproche de Sachs et de Gelman par son questionnement du silence de Dieu et par la douloureuse expérience de l'exil.

Territoires de mémoire se compose de trois parties : «Écritures de la perte : Le poème comme mémorial», « Écritures de l'exil : Le poème comme lieu de l'absence », et «Écritures de la mémoire: Dans le secret de la rencontre ». Dans les différents chapitres, l'auteure fait un admirable close reading des poèmes qu'elle met en rapport avec les notions d'absence, de mémoire, de survie, de deuil, de perte et de l'expérience juive de l'exil. Dans la première partie, l'auteure entreprend la tâche délicate de rencontrer les victimes évoquées dans les poèmes. Des questions comme « Comment représenter ceux qui sont morts et que l'on se refuse à oublier? » Comment éviter de les figer dans l'image stéréotypée de la victime, comment libérer la représentation des disparus de la négation de la mort?» (p. 31) servent de fil rouge. Taïeb met les poèmes en rapport avec les mémoriaux aux victimes de l'histoire comme celui de Berlin : le mémorial aux Juifs d'Europe assassinés, ou celui de Buenos Aires, le Parc de la mémoire, un espace consacré à la mémoire des victimes du terrorisme d'État.

Dans la deuxième partie,l'auteureétudieles poèmes et leur lien à la thématique de l'exil. Dans cette section, elle se concentre d'abord sur les poèmes de Sachs et Jabès pour y déceler la spécificité de la mémoire juive, pour se tourner ensuite vers les trois auteurs du corpus afin d'y retracer le rapport de l'exil à la mystique juive. Finalement, la troisième partie est vouée à la recherche d'un horizon commun vers lequel les « territoires de mémoires » convergent dans les poèmes. Dans cette partie Lucie Taïeb tente de montrer que « c'est la singularité même de la parole poétique et son éventuelle marginalité qui demeurent garantes de la transformation possible du poème - "dans le secret de la rencontre” - en territoire de mémoire. » (p. 395)

Le défi d'interpréter le langage poétique de trois auteurs qui évoquent une écriture de la violence comme « refuge » et « auberge » m'a rappelé une thèse soutenue à Amsterdam en 2004, Le génocide des Arméniens: La littérature de la mémoire par A. E. Kinébanian. L'auteur y donnait la parole à des écrivains arméniens d'expression française qui veulent reconstituer une mémoire des victimes du génocide de 1915 et de leurs descendants. Les victimes n'ont pas de sépulture et ont été doublement assassinées du fait que le génocide a été effacé de l'Histoire. L'écriture peut servir de médiation pour sortir de l'emprise du crime. Selon Kinébanian, la seule stratégie possible au survivant pour préserver du néant les victimes massacrées est de suspendre leur mort en «offrant son propre corps comme tombeau. » Un des chapitres de la première partie de Territoires de mémoire s'intitule «Le poème comme tombeau » (p. 95-147). En même temps, Lucie Taïeb se concentre sur d'autres métaphores et sur la possibilité pour le texte poétique de devenir « un espace d'accueil ». Elle étudie la représentation textuelle de trois auteurs exilés, endeuillés, éprouvant la difficulté d'écrire pour transmettre la mémoire des disparus. Ce n'est pas l'indicible qui l'intéresse, mais plutôt « la capacité du langage à recueillir la réalité », la possibilité pour le poème « d'héberger à la manière d'un mémorial, la mémoire collective de la Shoah. » (p. 12) L'auteure a choisi des textes qui ne sont pas des témoignages poétiques d'une expérience vécue des camps ou du ghetto, mais plutôt des ouvrages d'individus «à la fois en dehors de l'événement et intimement “concernés” par lui. » (p. 398)

L'essai de Taïeb offre un cadre interprétatif intéressant et aiguise notre sensibilité par la façon unique dont l'écriture poétique constitue un réceptacle pour la mémoire collective et individuelle de la violence historique.

Désirée Schyns 


\section{LES ENFANTS DU SILENCE. MEMOIRES D'ENFANTS CACHÉS}

Jean-Pierre Guéno

Toulouse, Milan, 2013, 87 p.

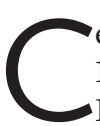

e livre est une adaptation pour enfants de 10 à 12 ans d'un ouvrage paru en 2002 aux éditions Librio et intitulé Paroles d'étoiles. Mémoire d'enfants cachés 1939-1945. Comme l'indique ce sous-titre de la première édition, il présente des récits - sous la forme, parfois, de lettres - d'enfants juifs cachés en France durant la Seconde Guerre mondiale et la déportation de leurs parents, dont la plupart ne sont jamais revenus.

D’enfants cachés, c'est-à-dire que l'on a cachés, avec les meilleures intentions du monde, mais presque toujours de force, contre leur volonté, après les avoir arrachés à leurs parents ou après que ceux-ci les ont éloignés d'eux. Avec pour résultat, au-delà d'une survie peut-être salutaire, une incompréhension douloureuse et quelquefois insurmontable. Cette souffrance valaitelle mieux que la mort, se demandent certains de ces rescapés, une mort, pour le moins, en compagnie de leurs parents, de leurs frères et sœurs ? La réponse est dans le mal-être qu'ils ont éprouvé pour le reste de leurs jours.

Les confessions des adultes que sont devenus ces enfants cachés peuvent-elles vraiment être lues par des enfants de 10-12 ans comme le prévoit Jean-Pierre Guéno dans sa préface (la quatrième de couverture annonce même un courageux « dès 10-1lans ») ? On peut en douter, bien qu'un lexique s'efforce de venir en aide aux lecteurs les plus démunis d'informations en matière de persécutions antijuives. La grande littérarité des récits, leurs nombreux sous-entendus en font certes des témoignages raffinés, mais difficilement appréciables dès lâge mentionné. On sent qu'ils n'ont pas été destinés prioritairement à des enfants et seule une lecture accompagnée, par un instituteur par exemple, pourrait les mettre à la portée d'un lectorat aussi juvénile, sans compter l'atrocité de certains vécus qui risquerait de troubler profondément des esprits encore fragiles.

En revanche, l'adulte que je suis, grand amateur, par ailleurs, de textes sur l'enfance, les a lus avec beaucoup d'émotion et, se souvenant d'une pensée de Theodor Herzl qui, dans son État des Juifs, affirmait qu' « après une courte période de tolérance, l'hostilité qui poursuit [les Juifs] finit toujours par se réveiller », l'adulte que je suis, donc, a frémi en lisant la réflexion d'une paysanne du Vercors qui avait pourtant caché une enfant juive pendant deux ans : lorsqu'elle annonça à la petite que la France était enfin libérée et qu'elle n'obtint qu'une muette stupéfaction en réponse à ce qu'elle considérait comme une nouvelle fracassante, la brave dame s'exclama : «Ces Juifs! Ils ne sont jamais contents ! »

«Après une courte période de tolérance... » Herzl avait-il vu juste?

Jean-Pierre Pisetta

$\bullet$

\section{LE MYTHE DE LA TRANSITION PACIFIQUE. VIOLENCE ET POLITIQUE EN ESPAGNE (1975-1982)}

\author{
Sophie Baby \\ Madrid, Casa de Velázquez, 2012, 527 p.
}

D 'emblée, le volume impressionne par sa taille : 527 pages, dont 440 d'un texte dense, complété par une imposante bibliographie et de précieux documents annexes. Il s'agit là du résultat éditorial d'une thèse dont l'envergure relève quasiment du doctorat d'État. Ce texte est d'ailleurs, comme le précise l'auteur dans les remerciements, l'aboutissement de plus de quinze années de travail, une dizaine pour la thèse à proprement parler, soutenue en 2006 à l'université Paris 1 Panthéon-Sorbonne, six de plus pour le processus d'édition du livre issu de ces travaux.

Comme son titre l'indique, la recherche porte sur la Transition politique en Espagne, entre la mort du dictateur Francisco Franco Bahamonde (novembre 1975) et l'année 1982, qui marque l'arrivée au gouvernement des élus socialistes (octobre). Sept années au cours desquelles, après une période de tâtonnements, est élaborée, votée et adoptée par référendum, le 6 décembre 1978, une Constitution démocratique, dans le cadre d'un régime monarchique conduit par Juan 
Carlos I, le dauphin de Franco. L'accession au pouvoir des socialistes à la fin de la période consolide le régime en permettant pour la première fois l'alternance politique dans ce nouveau cadre démocratique. Tout est donc bien qui finit bien et, jusqu’à la fin des années 1990, le discours dominant sur cette période de l'histoire du pays est que la Transition politique en Espagne s'est déroulée idéalement sur la base d'un consensus qui a permis aux acteurs politiques de s'entendre sur l'essentiel (la mise en place d'un régime démocratique), moyennant un certain nombre de renoncements, de part et d'autre. Une Transition modèle, qui servira de référent à de nombreux pays dans leur gestion de la sortie de dictatures, les nations latino-américaines dans les années 1980, puis les pays d'Europe de l'Est après la chute du mur de Berlin. Pour Sophie Baby, en revanche, ceci relève du «mythe » (terme utilisé pour la première fois par B. André Bazzana), c'est-à-dire d'un discours qui, répété à l'envi, a fini par acquérir un statut de vérité, au mépris de la réalité historique.

«Le mythe de la Transition pacifique », c'est précisément cela que les recherches de Sophie Baby tendent à déconstruire. Si, à la date de parution de cet ouvrage [2012], elle n'est ni la première ni la seule à le faire, en revanche, ce qui assurément fascine dans son travail, c'est sa manière très convaincante de mener la démonstration et le sérieux de sa démarche. Le mythe d'une Transition pacifique vise en réalité à en nier la violence, ce qui correspond à une fonctionnalité politique bien précise, assumée par les élites qui l'ont menée à bien, mais aussi sociale. Sophie Baby prend le parti, inverse, de faire émerger la violence politique au cours de la période. Son ouvrage dévoile alors la partie immergée d'un colossal iceberg. Méthodiquement, patiemment, courageusement, l'auteur a recensé, classé, analysé ce qu'elle regroupe sous la notion de «violences politiques en transition » (p. 15). Elle a réuni au total un corpus imposant - et jusque-là inédit par son ampleur - d'environ 3200 événements violents (avec plus de 700 morts) pour la période comprise entre la mort de Franco et la fin de l'année 1982, et qui selon l'auteur « ne constitue qu'un minimum » (p. 43). L'intérêt de ce corpus vient par ailleurs du fait qu'il ne se limite pas à l'expression « classique » de la violence politique (attentats), mais qu'il comporte également des actions multiformes qui ont entretenu un climat de violence constant tout au long de la période (menaces, émeutes, protestations, troubles dans l'espace public, etc.). Enfin, le corpus réunit aussi bien les manifesta- tions de violence de l'opposition que celles des agents de l'État, dans une spirale que rien ne semble pouvoir arrêter. Nous sommes loin de l'image canonique où la violence de la période est perçue soit comme un phénomène sporadique (le coup d'État manqué du lieutenant colonel Tejero le 23 février 1981), soit comme « une aberration périphérique qui rend encore plus éclatante la cohésion du reste de l’Espagne. » (p. 6)

Après une première partie liminaire qui dessine les contours des « violences politiques en transition » et offre un solide cadre épistémologique et méthodologique, l'ouvrage se structure en deux grandes parties, qui examinent successivement « Le cycle des violences protestataires » (p. 61-234) et « L’État et la violence » (p. 235-420). Le volume d'archives exploitées impressionne, tout comme les nombreux tableaux récapitulatifs, très éloquents, qui permettent de visualiser clairement les phénomènes observés. La clarté de la démonstration, tout autant que la justesse des analyses, rendent la lecture de cette somme passionnante. Et bien que l'objet d'étude appartienne désormais à un passé en apparence révolu, cette lecture de la transition résonne chez le lecteur contemporain, en écho à une actualité politique qui révèle clairement les limites du « mythe pacifique de la transition ». Si la plupart des souvenirs de la violence politique de la période, comme l'énonce Sophie Baby dans sa conclusion, « ont disparu de la mémoire collective, broyés sous le poids du mythe institutionnel» (p.438), cependant, le poids d'une transition inachevée et qui, à force d'occultation, n’a pas véritablement réglé ses comptes avec le franquisme, est aujourd'hui plus que jamais présent dans un pays où les démons du passé ressurgissent sans cesse, confirmant l'actualité de la conclusion de l'auteur : « La violence, en dépit des efforts déployés pour l'exclure de l'espace démocratique, est donc toujours au cœur du fait politique de l'Espagne contemporaine. » (p. 440) I

Nancy Berthier 


\section{RWANDA. RACISME ET GÉNOCIDE. L'IDÉOLOGIE HAMITIQUE}

Jean-Pierre Chrétien, Marcel Kabanda

Paris, Belin, 2013, 379 p.

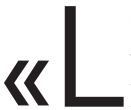

e propos n'est pas ici d'épancher une amertume, encore moins d'établir un sottisier », lit-on dans l'introduction du livre de JeanPierre Chrétien et Marcel Kabanda, deux historiens en place, mais était-il vraiment nécessaire de commencer cette étude en rabaissant le mérite de tous ceux « qui s’intéressent à cette région sans y avoir été, sans avoir respiré l'air des collines, sans avoir jamais été accueillis dans un enclos...? » Le catalogue de tous les manques de ces prétendus « faiseurs d'opinion » n'apporte malheureusement rien à la question.

Cela dit, l'essai n'en reste pas moins excellemment écrit et savamment élaboré. Un génocide ne s'effectue pas sur un coup de tête, ni pour venger un crash aérien, fût-il supposé être l'œuvre de la frange armée d'une population haïe. C'est d'abord la haine suscitée par cette population, haine ensuite instrumentalisée à des fins politiques, qui est le moteur de la tuerie et tout l'intérêt du travail de MM. Chrétien et Kabanda est de faire remonter cette exécration à des théories surprenantes et vieilles de plus d'un siècle d'âge.

L’idéologie hamitique ou chamitique qui serait à la source du racisme rwandais est née au milieu du XIX siècle et doit son appellation à Cham, fils de Noé rejeté par son père en raison d'un manque de respect. Selon les penseurs qui la véhiculent, les Hamites étaient « des Caucasoïdes pastoraux arrivés vague après vague, mieux armés et d'esprit plus vif que les agriculteurs nègres à peau sombre » (toutes les citations qui suivent sont tirées des observations ou des théories critiquées par les auteurs). L’Afrique noire aurait donc été colonisée, des siècles, voire des millénaires avant l'arrivée des Blancs, par des « races supérieures venues d'Asie » s'opposant aux « nègres en tant que tels », ces « milliers de gorilles dégrossis qui pullulent et végètent sur un sol pourtant plein de richesses et de ressources. »

Des Hamites ou Chamites, « tribu perdue d'Israël », descendant d'un Cham réprouvé par Noé: il n'en faudra pas plus pour qualifier de «Juifs africains » (ce n’est pas pour rien que les auteurs du présent ouvrage avaient pensé l’intituler «Du Juif au Tutsi ») ces gens «élancés aux traits fins et sympathiques. »

L'attrait esthétique constitue ainsi le premier lien entre les explorateurs blancs et ces noirs « nouveaux venus » qui se différencient des nègres « autochtones». « Il ne nous fallut qu'un instant, écrit John Hamming Speke en 1861, pour comprendre que les gens avec lesquels nous nous trouvions maintenant ne ressemblaient en rien aux grossiers indigènes des districts voisins. » Trente ans plus tard, Stanley renchérissait : « De beaux pasteurs s'occupent de leurs troupeaux et des négroïdes au nez épaté et à la peau très noire s'attaquent au sol avec leurs bêches. »

Sauf que cette belle prestance a son revers : adulés dans un premier temps parce que les Blancs se reconnaissent un tantinet en eux, ces êtres qui auraient pu être façonnés par le ciseau « d'un Praxitèle » ne se laissent pas - facilement du moins - mener par le bout du nez et leur résistance est bientôt associée à de la fourberie, association à laquelle cèdent volontiers, font remarquer à juste titre les auteurs, tous les colonisateurs. Les Hamites, « classe supérieure chez les Nègres », ont ainsi fait naître, à la fois, une fascination et une méfiance, et leur prétendue «supériorité», théorisée voire judaïsée par les Blancs, va être inoculée par ces derniers dans les rapports sociaux de cet endroit du monde, au point de passer, à la fin, pour la réalité.

Aussi, près d'un siècle avant le génocide desdits Hamites (appellation savante, donc, des Tutsi), les ingrédients qui vont le provoquer sont déjà en train de mijoter.

Les autorités ecclésiastiques prennent bientôt le relais des explorateurs et des idéologues ; les Tutsi sont christianisés prioritairement et deviennent les intermédiaires privilégiés entre le pouvoir blanc et le peuple rwandais, constitué, toutefois, en majorité de Hutu.

Quelques décennies plus tard, en 1959, à la veille de l'indépendance, une « Note sur l'aspect social du problème racial indigène au Rwanda », rédigée par des Hutu, est remise aux autorités coloniales. On y dénonce une double domination : celle des « Hamites » et celle de «l'Européen », cette dernière s'opérant de surcroît par le « canal » des mêmes Hamites.

Alors que le Congo est agité par une effervescence indépendantiste dontles leaders sont soupçonnés d'avoir des accointances «bolchéviques », l’Église change son fusil d'épaule et soutient les récriminations des Hutu, au grand dam des Tutsi qu'elle avait favorisés jusque-là. 
Ces tensions aboutissent aux affrontements raciaux de la Toussaint 1959, au cours desquels les Tutsi connaissent leurs premiers massacres et qui conduisent, après la « pacification » opérée par la Belgique, à leur éviction de tous les postes de pouvoir.

La première république, celle de Grégoire Kayibanda, voit le jour en 1962 dans un Rwanda où les Hutu, déjà majoritaires dans la population, le seront également dans toutes les instances dirigeantes pendant une trentaine d'années. La deuxième république, celle de Juvénal Habyarimana, perpétuera la racialisation de la société rwandaise inventée et institutionnalisée par les Blancs : le Tutsi est désormais, unanimement et même historiquement, considéré comme un - comme le seul - envahisseur.

MM. Chrétien et Kabanda brossent ainsi, par touches et couches successives, le portrait du génocide à venir et, dans les pages où il apparaît enfin dans toute son horreur, une impression de déjà vu nous saisit : le nettoyage du Rwanda par le Hutu Power ressemble en effet - par la méthode (systématique), par l'implication des populations locales (décentrées) qui n'y auraient sans doute pas participé si elles n'y avaient été poussées «del'extérieur», par l'indication raciale (et unilatérale) d'un ennemi - au nettoyage des pays de l'Est accompli par les Einsatzgruppen durant la Seconde Guerre mondiale. «Judenfrei » clamaient les tueurs nazis dès qu’ils pensaient avoir vidé une région de toute présence juive ; «Tutsifrei » espéraient manifestement pouvoir affirmer eux aussi ces anciens sujets de l'Allemagne (le Rwanda avait été une colonie allemande de 1890 à 1916).

On regrette cependant que le livre ne donne pas suffisamment la mesure des rétorsions qu'ont subies par la suite les Hutu, notamment ceux qui s'étaient réfugiés au Congo et qui n'étaient pas tous, loin de là, des génocidaires, sans compter que de nombreux Hutu favorables à un partage du pouvoir avec les Tutsi, avant le génocide, ont subi le même sort que ces derniers, ce que MM. Chrétien et Kabanda ne manquent toutefois pas de signaler dès le début du chapitre 9 intitulé $L e$ génocide (avril-juillet 1994) : les mots et les choses. Ils expliquent notamment: «Sont visés les Tutsi dans leur ensemble et des Hutu de l'opposition identifiés à des “complices”. » (p. 213) Et ce, même si les nombreux massacres de Hutu qui ont suivi le génocide des Tutsi ne peuvent sans doute pas - comme l'affirment les auteurs du livre - être considérés comme un second génocide.
Enfin, il ne suffit pas de ridiculiser des théories de complots américano-tutsi pour contester l'ingérence des États-Unis dans la région avant ou après le génocide : rappelons-nous le rôle qu'ils ont joué dans l'assassinat de Patrice Lumumba et dans la prise du pouvoir, par Mobutu, dans le Congo voisin pour qu'il apparaisse clairement qu'ils ne pouvaient pas ne pas s'intéresser de près à ce qui se passait au Rwanda. La lutte contre les affinités gauchistes présumées des Tutsi qu'avait menée l'Église catholique aux côtés des Hutu dans les années qui précédaient l'indépendance se devait d'être cautionnée et poursuivie - comme partout ailleurs dans le monde - par les autorités du Nouveau Monde.

Cela n'enlève rien à la qualité de cet ouvrage qui démontre de façon exemplaire que le racisme - en d'autres termes la peur de ceux qui nous sont étrangers - fait corps avec le genre humain et qu'un savant discours peut, avec le temps et beaucoup de détermination, rendre ce regard porté sur l'autre prépondérant dans les rapports sociaux. Les nazis y étaient parvenus avec les Juifs ; les Hutu y sont parvenus avec les Tutsi. I

Jean-Pierre Pisetta

\section{CIEL AVEC TROU NOIR}

\author{
Caroline Alexander \\ Bruxelles, MEO, 2014, 240 p.
}

l s'agit du récit de vie d'une petite juive allemande née au-dessus d'un lupanar. Sa mère parvient à la faire sortir de l'Allemagne nazie à temps, tandis qu'ellemême et son fils disparaissent dans l'enfer concentrationnaire. Certains affirmeront : « encore un livre sur ce sujet ». Pierre Mertens leur répond dans une remarquable préface qui nous livre ses réflexions sur l’indicible, la « déferlante de manuscrits », le pardon, l'oubli...

Le récit de Caroline Alexandre est effectivement émouvant. Grâce à un patchwork de courts chapitres de deux ou trois pages, parfois même d'une demie, par petites touches, quasiment du pointillisme, avec beaucoup de franchise et de pudeur, une légèreté et une élégance d'écriture remarquables, l'auteur nous fait partager son expérience. La petite ville où elle est 
née, devenue «judenrein », a invité en 1989, pour ledit « travail de mémoire », des anciens habitants juifs miraculés, résidant sur les cinq continents. Cela a été, pour l'auteur, l'occasion de rappeler l'intégration des Juifs allemands et leur apport à l'économie, les amis et relations exprimant leur antisémitisme, le nazisme au quotidien, mais aussi la folle paranoïa des SS fusillant les soldats revenus au pays suite à un échange de prisonniers, car considérés comme déserteurs. Émouvants sont aussi le rappel permanent de scènes vécues avec sa mère et son frère aîné, et la recherche pathétique du lieu de leur assassinat. Il ne s'agit pas d'un livre sur Auschwitz, mais sur une vie marquée par Auschwitz. Un livre que l'on quitte avec regret.

Henri Goldberg

\section{VIVRE C'EST VAINCRE. J'AI ÉTÉ TÉMOIN DE L'HOLOCAUSTE}

Général André Rogerie

Maulévrier, Hérault, «L'écho de leurs voix », 1988, 106 p.

A ndré Rogerie a 21 ans en 1943 quand il prépare l'examen de Saint Cyr. C'est alors qu'il décide de rejoindre la France Libre du général de Gaulle par l'Espagne. Mais arrêté à Dax, il est interné dans différentes prisons françaises et finalement déporté, fin octobre 1943, dans les camps de Buchenwald, puis Dora, Lublin et finalement à Auschwitz-Birkenau. De là, début 1945, il fait les marches de la mort, il passe ainsi par de nouveaux camps : Groß-Rosen, Nordhausen, à nouveau Dora. Enfin Harzungen, d'où il s'évade en avril 1945 dans la région de Magdebourg pour trouver refuge parmi des prisonniers de guerre français. Deux mois plus tard, il est rapatrié et, de mémoire, il se met à rédiger ce témoignage, J'ai été témoin de la Shoah, qu'il publie à compte d'auteur. En 1988, réagissant aux allégations des négationnistes sur le génocide des Juifs, ce catholique pratiquant et général de l'armée française réédite son ouvrage. Décédé en 2014, une seconde réédition vient de paraître.

Ce témoignage retient l'attention à deux titres : c'est le témoignage d'un non juif sur le judéocide à Birkenau et, surtout, il entre en lutte contre l'argumentation fallacieuse des négationnistes qui comptent dans leurs rangs le fameux Faurisson tentant, lors de la première réédition, de discréditer l'ouvrage sur son blog « inofficiel ».

André Rogerie a séjourné au camp hôpital de Birkenau (réservé aux non Juifs, car les Juifs étaient gazés dès qu'ils étaient déclarés inaptes au travail). De ce camp hôpital, on voyait un crématoire. Il est d'ailleurs curieux que Faurisson ne conteste pas la description que livre André Rogerie des cortèges de Juifs et de Tziganes dirigés vers ce crématoire, évoquant la sordide odeur de viande brûlée qui envahissait le camp. Faurisson se contente d'écrire : «il n'a pas le droit d'écrire: J'ai assisté à la Shoah à Birkenau », comme si Rogerie s'exprimait en son nom. Pourtant, ce dernier signale qu'il reprend le témoignage d'un déporté travaillant dans le « kommando » chargé de détruire cette installation, et que le schéma qu'il en fait dans son ouvrage est correct. Par contre, Faurisson ne conteste pas les dires de Rogerie concernant son séjour au camp hôpital où il était correctement nourri et soigné, il écrit même : "ce qui est vrai en revanche».

Un autre révisionniste notoire, Vincent Reynouard, extrait dans le témoignage d'André Rogerie : « je suis emmené dans le camp des femmes pour passer sous l'appareil de rayons $X$, car rien ne manque à Birkenau ». Ce à quoi Rogerie répond de la façon suivante : « ce fait, je ne l'ai donc ni inventé, ni puisé dans un livre révisionniste. Je l'ai trouvé dans le témoignage d'un ancien déporté... » Il nous faut également ajouter qu’à la suite de son témoignage, on peut lire: «il a été apporté [parlant de l'appareil de rayons $\mathrm{X}$ ] ici pour essayer de nouvelles méthodes de stérilisation. Les essais ont été tentés sur de jeunes Juifs qui ont été entièrement brûlés aux points d'application ». Cette question n'intéresse évidemment pas le négationniste qui n'y voit certainement qu'un « point de détail »!

Henri Goldberg 